\title{
SYMPOSIUM
}

\section{Functional brain organization for visual search in ASD}

\author{
BRANDON KEEHN,,${ }^{1,2}$ LAURIE BRENNER, ${ }^{1}$ ERICA PALMER,${ }^{1}$ ALAN J. LINCOLN, ${ }^{3}$ \\ AND RALPH-AXEL MÜLLER ${ }^{1,4}$ \\ ${ }^{1}$ Brain Development Imaging Laboratory, Department of Psychology, San Diego State University, San Diego, California \\ ${ }^{2}$ Joint Doctoral Program in Language and Communicative Disorders, San Diego State University, San Diego, California, \\ University of California, San Diego, San Diego, California \\ ${ }^{3}$ Developmental Neuropsychology Laboratory, Alliant International University, San Diego, California \\ ${ }^{4}$ Department of Cognitive Science, University of California, San Diego, San Diego, California
}

(Received December 11, 2007; Final Revision July 22, 2008; Accepted July 22, 2008)

\begin{abstract}
Although previous studies have shown that individuals with autism spectrum disorder (ASD) excel at visual search, underlying neural mechanisms remain unknown. This study investigated the neurofunctional correlates of visual search in children with ASD and matched typically developing (TD) children, using an event-related functional magnetic resonance imaging design. We used a visual search paradigm, manipulating search difficulty by varying set size $(6,12$, or 24 items), distractor composition (heterogeneous or homogeneous) and target presence to identify brain regions associated with efficient and inefficient search. While the ASD group did not evidence accelerated response time (RT) compared with the TD group, they did demonstrate increased search efficiency, as measured by RT by set size slopes. Activation patterns also showed differences between ASD group, which recruited a network including frontal, parietal, and occipital cortices, and the TD group, which showed less extensive activation mostly limited to occipito-temporal regions. Direct comparisons (for both homogeneous and heterogeneous search conditions) revealed greater activation in occipital and frontoparietal regions in ASD than in TD participants. These results suggest that search efficiency in ASD may be related to enhanced discrimination (reflected in occipital activation) and increased top-down modulation of visual attention (associated with frontoparietal activation). (JINS, 2008, 14, 990-1003.)
\end{abstract}

Keywords: Autism, Asperger's disorder, fMRI, attention, Response time, Visual perception

\section{INTRODUCTION}

Individuals diagnosed with autism spectrum disorder (ASD) exhibit elementary abnormalities of attention and perception (see Dakin \& Frith, 2005, for review). Atypical visuospatial processes often manifest as areas of strength and include superior performance on the Embedded Figures Test (EFT; Jarrold et al., 2005; Jolliffe \& BaronCohen, 1997; Morgan et al., 2003; Shah \& Frith, 1983), the Wechsler block design (Caron et al., 2006; Shah \& Frith, 1993), and visual search tasks (O'Riordan, 2004;

Correspondence and reprint requests to: Ralph-Axel Müller, Department of Psychology, San Diego State University, MC1863, 6363 Alvarado Ct. \#225E, San Diego, CA. E-mail: amueller@ sciences.sdsu.edu
O'Riordan et al., 2001; Plaisted et al., 1998). Although previous studies have shown that individuals with autism excel at visual search, the brain bases for the advantage seen in ASD remain unknown. Understanding the neural mechanisms of processing strengths, such as visual search, may provide a window onto atypical profiles of sensory and cognitive processing and thus help elucidate the primary disturbances of functional brain organization in individuals with autism.

Visual search paradigms require participants to determine the presence or absence of a target item located within an array of distractors. Visual attention is guided within the array by bottom-up and top-down mechanisms (see Wolfe, 1994, for discussion). Bottom-up modulation of visual attention is dependent on the physical characteristics of the stim- 
uli; the level of bottom-up stimulation is determined by differences between physical properties of the target and surrounding distractors. In contrast, top-down processes modulate bottom-up signals based on task objectives and the goals of the participant. Search difficulty is dependent on factors such as the number of items within the array (set size) and target-distractor or distractor-distractor similarity. Search efficiency is measured as the slope ( $\mathrm{ms} /$ item) of the response time (RT) by set size function. Efficient search is represented by a relatively flat RT by set size slope $(<10$ $\mathrm{ms} /$ item) and indicates that all items within the array are processed in parallel. Conversely, steeper slopes are indicative of more inefficient search processes, which require serial scanning of individual items. As similarity between distractor types increases (or if distractors are homogeneous), search for the target becomes less dependent on set size, and proceeds in an efficient, parallel manner. Alternatively, as similarity between target and distractors increases the saliency of the target decreases, augmenting the difficulty of search as set size increases and resulting in inefficient, serial search (Duncan \& Humphreys, 1989).

Visual search relies on a network of brain areas that orient visual attention, filter irrelevant distractors, plan and execute eye-movements, and identify objects at the locus of attention. Functional neuroimaging studies of adults have revealed a widespread system of cortical and subcortical structures necessary for visual search (see Kastner \& Ungerleider, 2000, for review). While bottom-up, sensory-driven mechanisms of visual attention are mediated by visual cortices, top-down guidance relies on a frontoparietal network (Corbetta \& Shulman, 2002). The posterior parietal lobe is in part responsible for representing and selecting spatial locations required for visual search (Donner et al., 2000; Gitelman et al., 2002; Leonards et al., 2000; Müller et al., 2003; Nobre et al., 2003; Wilkinson et al., 2002), whereas attentional guidance and target selection are modulated by the frontal eyes fields (FEF; Muggleton et al., 2003). Additionally, the prefrontal cortex may also play a role in difficult (i.e., inefficient) searches (Anderson et al., 2007). While this role remains to be fully characterized, Anderson and colleagues hypothesize that it may relate to controlling working memory necessary for selective attention.

Although the neural correlates of visual search processes are well understood in the adult brain, the corresponding developmental literature remains very limited. Booth and colleagues (2003) found similar patterns of frontal and parietal activation between children 9 and 12 years old and adults on a color-form conjunction visual search task. A recent cross-sectional study of individuals from 8 to 20 years of age found that right hemisphere dominance for visual search increased with age in frontal and parietal regions (Everts et al., 2008). Thus, a network of frontal and parietal regions is recruited during visual attention tasks in children and adults, although the developmental changes associated with this network are not well defined.

Studies investigating visual search in ASD have revealed consistently accelerated RTs compared with typically devel- oping (TD) individuals, with the largest RT advantage occurring for target absent trials and trials with larger set sizes. While the greatest ASD advantage often occurs in the hardest search trials, the absence of group differences in RT on target present trials and smaller set sizes may be due to ceiling effects, as increasing difficulty of feature search (i.e., when target and distractors differ with respect to only one stimulus feature, such as orientation) has yielded more robust group differences. O'Riordan and colleagues (2001) examined visual search abilities in children with ASD using both easy (efficient) and hard (inefficient) feature search conditions, in which participants searched for either a tilted line in an array of vertical distractors (easy condition) or a vertical line among tilted distractors (hard condition). While there was no difference between TD and ASD children on the easy search task, the ASD group was significantly faster than the TD group in the hard condition. Furthermore, the ASD group evidenced shallower RT by set size slopes compared with the TD group for both conditions, indicating the cost of additional distractors was greater in TD as compared to ASD participants. The authors hypothesized that accelerated RT and reduced slope by individuals with ASD may be the result of enhanced discrimination of stimulus items and/or superior top-down modulation of excitatory and inhibitory mechanisms.

To determine whether superior ASD visual search abilities are derived from enhanced discrimination, O'Riordan and Plaisted (2001) used a color-form conjunctive search task, in which the target shares a feature with each set of distractors (e.g., target was red " $F$ " and distractors were red "E" and green "F"), to examine the effect of target-distractor similarity on RT. While increasing target-distractor similarity resulted in longer RTs for both ASD and TD participants, the TD group was slowed to a greater extent than the ASD group, which suggests that search advantage in autism is related to enhanced visual discrimination.

To assess whether enhanced discrimination was due to superior top-down modulation of visual attention, O'Riordan (2000) examined whether individuals with ASD achieve their advantage via enhanced distractor inhibition and/or target excitation. Using a color-form conjunction the authors varied either the identity of the target (excitation of target features) or distractors (inhibition of distractor features) to examine the degree to which excitation and inhibition of object features facilitates search. While previous findings of accelerated search were replicated, the magnitude of object-based positive and negative priming effects was equivalent for both groups, suggesting that accelerated RT for individuals with ASD was not the result of greater top-down modulation of object-based representations. However, it remains undetermined whether enhanced discrimination in ASD is achieved via augmented modulation of bottom-up, lower-level perceptual processing.

Although the current study is the first fMRI investigation of visual search in ASD, previous studies have examined neurofunctional differences associated with EFT performance (Lee et al., 2007; Manjaly et al., 2007; Ring 
et al., 1999). On this task, TD individuals recruited a network of frontal (Lee et al., 2007; Ring et al., 1999) and parietal (Lee et al., 2007; Manjaly et al., 2007; Ring et al., 1999) regions. In contrast, individuals with ASD showed patterns of activation restricted to more posterior areas in right occipital and left superior parietal lobe (Lee et al., 2007; Ring et al., 1999), and bilateral occipital cortex and cerebellum (Manjaly et al., 2007). These results suggest that superior EFT performance by individuals with ASD may be the result of enhanced lower-level perceptual processes.

The present study investigated the neurofunctional correlates of visual search in children and adolescents with ASD and a TD comparison group using an event-related fMRI design. Specifically, we manipulated distractordistractor similarity in a feature visual search task to examine the differences in efficient (homogeneous distractors) and inefficient (heterogeneous distractors) search processes. Attentional modulation in homogeneous distractor composition trials should be efficient as saliency of the target is increased when surrounding distractors are in the same orientation. Alternatively, heterogeneous distractor composition should be associated with inefficient, serial search, as distractors of different orientation reduce target salience. Based on previous behavioral and fMRI studies examining visual attention, we hypothesized that performance (accuracy, RT) would be superior in ASD as compared to TD participants and that this group difference would be more pronounced for heterogeneous than for homogenous distractor composition. We further expected neurofunctional differences, with greater activity in ASD as compared to TD children in posterior occipito-temporoparietal regions, to be interpreted as enhanced bottom-up processing. A further open question was whether performance in ASD would be primarily bottom-up, that is, whether activity in frontal regions would be reduced in comparison with TD children.

\section{METHODS}

\section{Participants}

Nine right-handed children and adolescents with ASD (all males), all of whom met DSM-IV-TR (APA, 2000) criteria for autism spectrum disorder, and an age-, nonverbal IQ-, and handedness-matched comparison group of 13 righthanded typically developing (TD) children and adolescents (all males) were included in the present study. The final ASD sample of nine was obtained from an initial sample of 13; four children with ASD were excluded from the final sample due to excessive movement during fMRI scanning. Clinical diagnoses were confirmed using the Autism Diagnostic Interview-Revised (ADI-R; Rutter et al., 2003), module 3 of the Autism Diagnostic Observation Schedule (ADOS; Lord et al., 1999), and expert clinical judgment (author AJL and Dr. Natacha Akshoomoff). According to
Table 1. Participant characteristics

\begin{tabular}{lcc}
\hline \hline & Autism $(n=9)$ & Comparison $(n=13)$ \\
& Range & $M(S D)$ \\
& $15 ; 1(2 ; 6)$ & $14 ; 11(2 ; 11)$ \\
& $10 ; 10-17 ; 11$ & $8 ; 2-19 ; 1$ \\
Age & $109(15)$ & $116(10)$ \\
Verbal IQ & $79-128$ & $103-133$ \\
& $110(20)$ & $112(11)$ \\
Nonverbal IQ & $70-140$ & $99-129$ \\
& & \\
ADOS Algorithm Score & $4(1)$ & \\
Communication & $2-6$ & \\
& $8(3)$ & \\
Social interaction & $4-13$ & \\
& $0(1)$ & \\
Repetitive behavior & $0-2$ & \\
ADI Algorithm Score & $19(1)$ & \\
Communication & $18-20$ & \\
& $23(3)$ & \\
Social interaction & $19-26$ & \\
Repetitive Behavior & $7(3)$ & \\
& $3-9$ & \\
\hline \hline
\end{tabular}

their ADOS scores, 7 children met criteria for a diagnostic classification of autism, one met criteria for a less severe classification of autism spectrum disorder, and one met ADOS criteria for autism in the social domain, but was below threshold in the communication domain. The latter child met full criteria for autism on the ADI-R, and was therefore included in the sample. Children with autismrelated medical conditions (e.g., Fragile-X syndrome, tuberous sclerosis) were excluded.

Participants in the TD group had no reported personal or family history of autism and were confirmed via parent report to be free of autism-related symptoms or any other neurological or psychiatric conditions. Independent-samples $t$ tests confirmed that the final ASD $(n=9)$ and TD $(n=13)$ groups were matched on age, $t(20)=0.1 ; p=.93$ and nonverbal IQ, $t(17)=-0.2 ; p=.83$, as determined using the Wechsler Abbreviated Scale of Intelligence (WASI; Wechsler, 1999; see Table 1). Informed consent was obtained from all participants in accordance with the University of California, San Diego and San Diego State University Institutional Review Boards.

\section{Experimental Task}

The target was an upright letter " $\mathrm{T}$ " and distractors were Ts rotated in three cardinal orientations $\left(90^{\circ}, 180^{\circ}, 270^{\circ}\right)$. Each target or distractor subtended a visual angle of $.35^{\circ}$ to $.38^{\circ}$ in both dimensions, depending on orientation. In the homogeneous condition, all distractors appeared in only one orientation, while in the heterogeneous condition distractors were presented in all three possible orientations (Fig. 1). Visual stimuli were presented in black on a light blue back- 


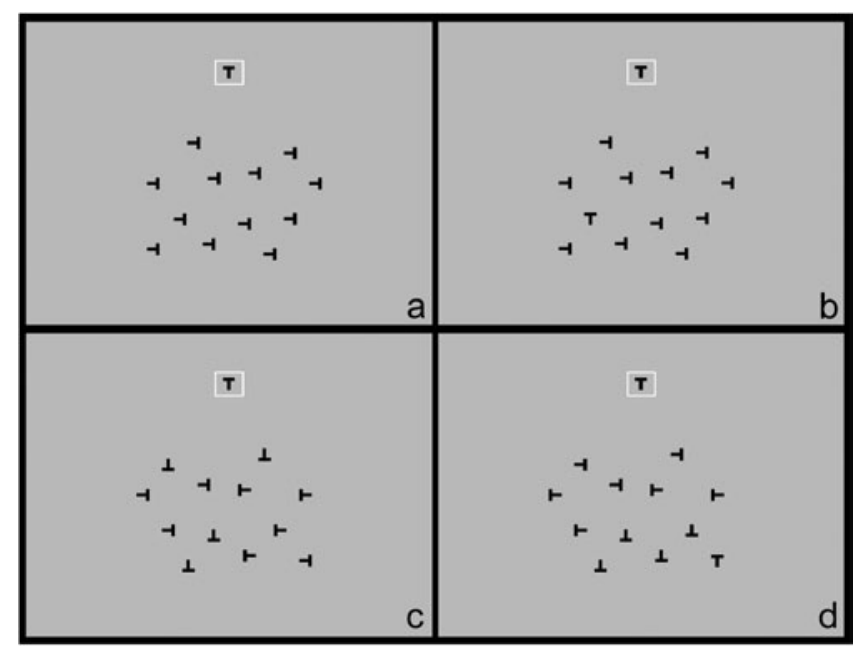

Fig. 1. Illustration of target absent (a) and target present (b) homogeneous, and target absent (c) and target present (d) heterogeneous search trials. Light blue background is represented in gray.

ground. The search arrays contained 24 possible stimulus locations. Each trial consisted of 6, 12, or 24 stimulus elements that were pseudorandomly arranged at specific array locations subtending $3.7^{\circ}$ by $2.7^{\circ}, 5.2^{\circ}$ by $3.7^{\circ}$, and $9^{\circ}$ by $4.5^{\circ}$, respectively, for each set size. Distractors were equally distributed on each side of the array, and the target appeared with equal probability on each side of the midline. In half of the trials, a target appeared within the search array (target present trials). In the remaining target absent trials, only distractors were presented. There were thus 12 trial types distinguished according to distractor composition, set size, and target presence. Additional baseline trials consisted of a solitary target (target present baseline) or a single distractor (target absent baseline), displayed in the center of the screen. Twenty trials of each condition were presented for a total of 280 trials. To reduce working memory demands, a target exemplar ("T") always appeared above the search array.

The experimental task was to indicate via a two-choice button box response whether the target stimulus was present or absent. A trial began with a fixation cross ("+") presented alone for $300 \mathrm{~ms}$. Next, with the fixation cross removed, the search array appeared and remained on the screen for $2200 \mathrm{~ms}$. Null trials, presenting a fixation cross only, were used for temporal jittering.

The experiment consisted of four runs, each with 70 search trials and 58 null trials. Within each run, trial types were presented in an optimized pseudorandom sequence created using RSFgen (AFNI; http://afni.nimh.nih.gov). Before the scanning session, a demonstration was given and practice trials were administered with corrective feedback. Participants were instructed to respond as quickly as possible without making errors.

Before collection of MRI data participants completed an initial visit, which included neuropsychological and diag- nostic testing, collection of behavioral data for the visual search experiment, and a mock scanning session. Behavioral data were collected to ensure participants could complete the task while in the scanner and to assess differences in performance due to the scanner environment. The purpose of the mock scanning session was to acclimate participants to the scanner environment (e.g., loud noise, enclosed space) and to practice remaining motionless.

\section{MRI Data Acquisition}

Imaging data were acquired at the Center for Functional Magnetic Resonance Imaging at the University of California, San Diego using a GE 3 Tesla HD Signa Excite scanner with an 8-channel head coil. High-resolution anatomical images were acquired using a standard FSPGR T1-weighted sequence. Each of the four functional runs was 5:10 min long, containing 128 whole-brain volumes acquired in 40 interleaved slices using a single-shot, gradient-recalled, echoplanar pulse sequence (TR $2500 \mathrm{~ms}$; TE $30 \mathrm{~ms}$; flip angle $90^{\circ}$; matrix $64 \times 64$; slice thickness $3.2 \mathrm{~mm}$; in-plane voxel size $3.4 \times 3.4 \mathrm{~mm}$ ). Participants' heads were stabilized with foam padding to reduce motion. The experiment was presented on a Pentium III $1.7 \mathrm{GHz} / 512 \mathrm{MB}$ laptop PC using Presentation software (www.neurobs.com). Behavioral responses were recorded using an MRI compatible response box. Participants viewed stimuli displayed on a backprojection screen at their feet using a mirror attached to the head coil.

\section{Data Analysis}

\section{fMRI data analysis}

Data were analyzed using the Analysis of Functional Neuroimages (AFNI; Cox, 1996). For each participant, the first four volumes of each run were discarded to remove signal equilibration effects. Each volume was slice-time and motion corrected. The four runs were then concatenated to create a single time-series with 496 volumes and smoothed with a Gaussian filter (FWHM $=6 \mathrm{~mm}$ ). Time points with excessive motion (greater than $2 \mathrm{~mm}$ ) were censored.

The hemodynamic response function for each stimulus type was estimated using a general linear model that included separate regressors to estimate the blood-oxygen-level dependent (BOLD) response at the onset of each stimulus and at each of the next 6 time-points ( $0-15 \mathrm{~s}$ poststimulus onset). Impulse response functions (IRFs) were estimated across time points 2 through 4 (5-10 s). A multiple regression analysis was performed on the estimated IRFs and the stimulus time series. The six motion parameters corresponding to translation and rotation were used as orthogonal regressors. Activation maps were normalized into Talairach space using AFNI auto-Talairach procedures and interpolated to $3 \mathrm{~mm}^{3}$ isotropic voxels.

One-sample $t$ tests were used to assess within-group differences for homogeneous and heterogeneous trials (sepa- 
rately and combined); additional two-sample independent $t$ tests were used to compare groups. A minimum cluster size of $513 \mathrm{~mm}^{3}$, a voxel connectivity distance of $5.82 \mathrm{~mm}$, and a single voxel threshold of $t(8) \geq 3.827 ; p<.005$ (ASD within-group), $t(12) \geq 3.424 ; p<.005$ (TD within-group), and $t(21) \geq 3.151 ; p<.005$ (group comparisons) was used to correct for multiple comparisons. Pair-wise comparisons were used to compare differences between homogeneous and heterogeneous trials within each group. For these comparisons, a minimum cluster size of $756 \mathrm{~mm}^{3}$, a voxel connectivity distance of $5.82 \mathrm{~mm}$, and a single voxel threshold of $t(8) \geq 3.354 ; p<.01$ (ASD group) and $t(12) \geq 3.054$; $p<.01$ (TD group) was used. All cluster corrections yielded a corrected threshold of $p<.05$, as determined by Monte Carlo simulation (AFNI program AlphaSim; Forman et al., 1995).

\section{Behavioral data analysis}

A mixed-model analysis of variance (ANOVA) with the factors group (ASD, TD), distractor composition (homogeneous, heterogeneous), target presence (absent, present), and set size $(6,12,24)$ was conducted on median RT for correct trials. The slopes and intercepts of the median RT $\times$ set size functions were calculated for target present and absent trials in both homogeneous and heterogeneous conditions. Slope is a measure of search efficiency, reflecting the RT cost of each additional distractor. Intercept of the $\mathrm{RT} \times$ set size function is associated with nonsearch, perceptual components related to the task such as early visual processing (Sternberg, 1966). Data were analyzed using SPSS 14.0. Partial eta-squared $\left(\eta_{\mathrm{p}}^{2}\right)$ is reported as a measure of effect size. Error bars in the figures represent one standard error of the mean.

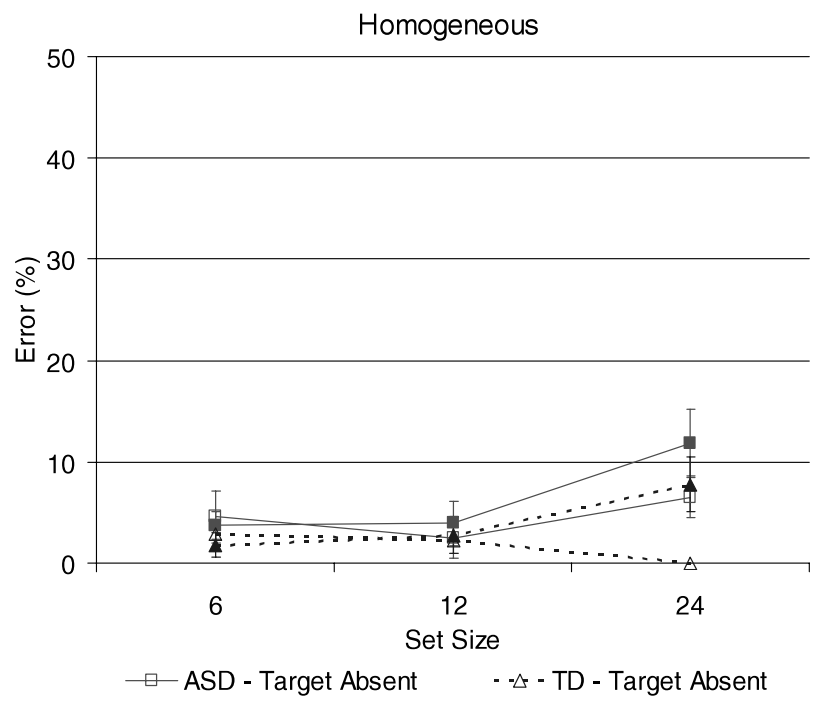

\section{RESULTS}

\section{Behavioral Results}

Behavioral response time measures were successfully collected during acquisition of fMRI data for 8 of the 9 ASD participants and 12 of the 13 TD participants; data from 2 participants were lost due to equipment malfunction.

\section{Error Rates}

Error rates were greater in heterogeneous compared with homogeneous trials, $F(1,18)=36.6 ; p<.001 ; \eta_{\mathrm{p}}^{2}=.63$, greater in present compared with absent trials, $F(1,18)=$ 22.6; $p<.001 ; \eta_{\mathrm{p}}^{2}=.56$, and increased with set size, $F(2,17)=22.4 ; p<.001 ; \eta_{\mathrm{p}}^{2}=.72$. There was no difference between groups in error rate, $F(1,18)=2.0$, nor were there any interaction effects between group and other factors. Correlational analyses between error rates and median RTs for each condition revealed no evidence of a speedaccuracy tradeoff for either group. Task compliance was confirmed, as mean error rates were $<24 \%$ in each participant, and thus no individuals were excluded based on error rate. See Figure 2.

\section{Reaction Time}

As illustrated in Figure 3, median RT was longer in heterogeneous than homogeneous trials, $F(1,18)=131.7 ; p<$ $.001 ; \eta_{\mathrm{p}}^{2}=.88$, longer in target absent than target present trials, $F(1,18)=90.4, p<.001, \eta_{\mathrm{p}}^{2}=.83$, and increased as a function of set size, $F(2,17)=67.6 ; p<.001 ; \eta_{\mathrm{p}}^{2}=$ .88. There was no main effect of group, $F(1,18)=0.0$, but there was a marginally significant group $\times$ distractor com-

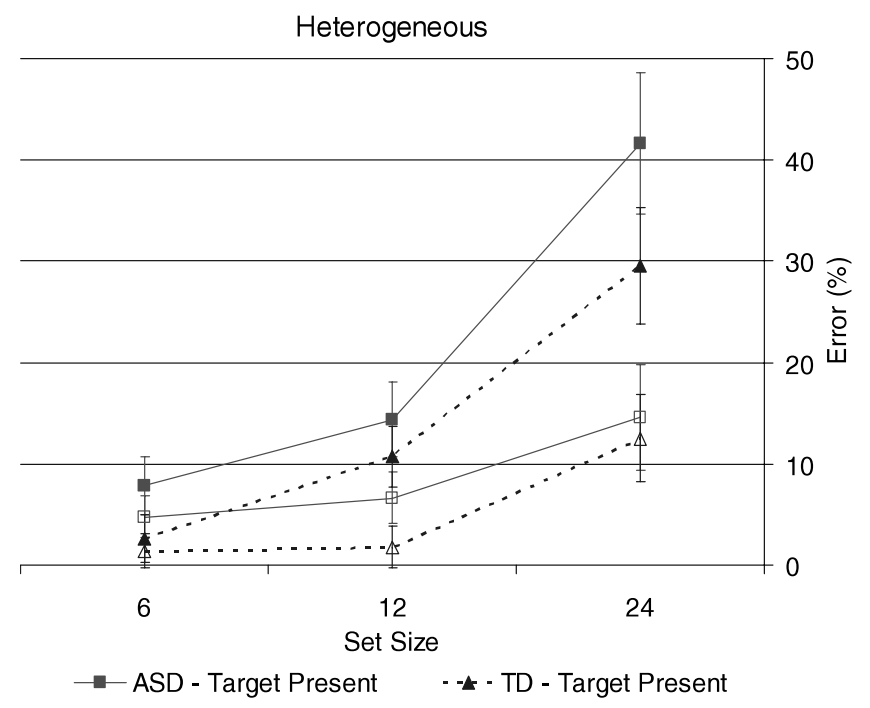

Fig. 2. Mean error rate as a function of group, distractor composition, target presence, and set size. Error bars represent standard errors of means. 

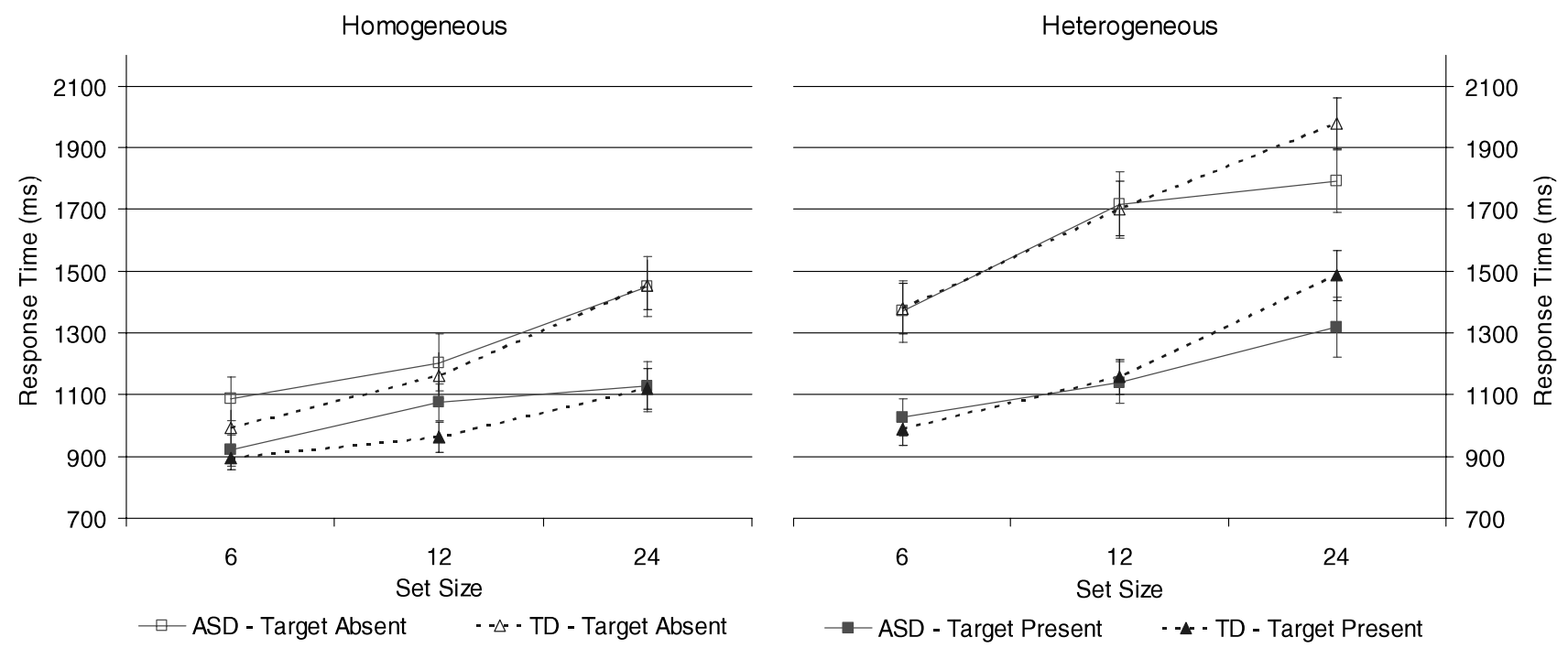

Fig. 3. Median RT for correct trials only as a function of group, distractor composition, target presence, and set size. Error bars represent standard errors of means.

position interaction, $F(1,18)=3.7 ; p<.07 ; \eta_{\mathrm{p}}^{2}=.17$, as individuals with ASD were slower than TD individuals in the homogeneous condition but faster in the heterogeneous condition compared with TD individuals. However, follow-up ANOVAs performed separately for homogeneous and heterogeneous trials revealed no significant group differences $(F \mathrm{~s}<1)$. Separate ANOVAs performed on each group showed a significant main effect of distractor composition for the TD comparison group, $F(1,11)=$ $77.9 ; p<.001 ; \eta_{\mathrm{p}}^{2}=.88$, and the ASD group, $F(1,7)=$ $123.4 ; p<.001 ; \eta_{\mathrm{p}}^{2}=.95$. In addition, there was a significant group $\times$ set size interaction, $F(1,18)=3.4 ; p<.03$; $\eta_{\mathrm{p}}^{2}=.34$, as individuals with ASD were less affected by larger set size compared with TD individuals. A separate ANOVA was used to compare baseline trials. Whereas there was no group difference in baseline target absent trials, $F(1,18)=1.3 ; p>.3 ; \eta_{\mathrm{p}}^{2}=.07$, TD individuals demonstrated significantly faster RT than individuals with ASD in the baseline target present trials, $F(1,18)=5.2 ; p<$ $.04 ; \eta_{\mathrm{p}}^{2}=.22$.

Slopes and intercepts of the RT $\times$ set size functions for the target-present and target-absent trials from the homogeneous and heterogeneous conditions were extracted from the median RT data reported above. Slopes were steeper, $F(1,18)=10.9 ; p<.005 ; \eta_{\mathrm{p}}^{2}=.38$, and intercepts were higher, $F(1,18)=39.9 ; p<.001 ; \eta_{\mathrm{p}}^{2}=.69$, for heterogeneous than homogeneous trials. Slopes, $F(1,18)=16.2$; $p<.001 ; \eta_{\mathrm{p}}^{2}=.47$, and intercepts, $F(1,18)=36.3 ; p<$ $.001 ; \eta_{\mathrm{p}}^{2}=.67$, were also greater in target absent as compared to target present trials. As illustrated in Figure 4, between-group analysis of RT $\times$ set size slope revealed a marginally significant effect of group, $F(1,18)=3.8 ; p<$ $.07 ; \eta_{\mathrm{p}}^{2}=.17$. Separate ANOVAs performed on each group showed a significant main effect of distractor composition, $F(1,11)=16.0 ; p<.005 ; \eta_{\mathrm{p}}^{2}=.59$, for the TD comparison group, but no main effect for distractor composition, $F(1,7)=1.1 ; p>.3 ; \eta_{\mathrm{p}}^{2}=.14$, for the ASD group. There was no difference between groups (ASD: 1018; TD: 955) for intercepts, $F(1,18)=1.1$, nor were there any interaction effects between group and other factors.

\section{fMRI Results}

Presentation of activation effects will be primarily limited to two comparisons: baseline trials versus fixation and search trials versus baseline. In many cases, results for the latter comparison did not differ substantially from those for the comparison of search trials versus fixation. However, because significant group differences in RT to baseline target present trials were observed, we also present findings for the comparison of search versus fixation whenever corresponding effects were not detected for the comparison with baseline trials.

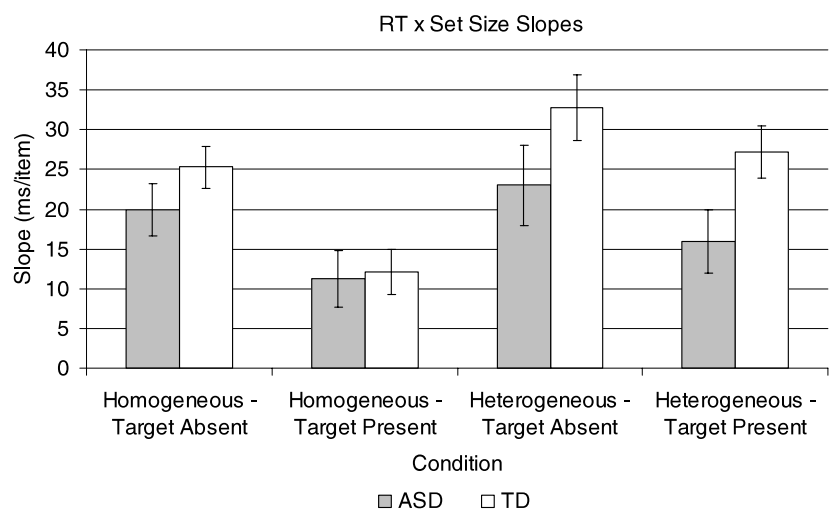

Fig. 4. RT $\times$ set size slope for ASD and TD group as a function of distractor composition and target presence. 
Table 2. fMRI BOLD activation for baseline trials versus fixation

\begin{tabular}{|c|c|c|c|c|c|c|c|}
\hline & \multirow{2}{*}{$\begin{array}{c}\text { Peak location } \\
\text { Additional regions } \\
(\% \text { volume of cluster })\end{array}$} & \multirow[b]{2}{*}{ Hemisphere } & \multicolumn{3}{|c|}{ Talairach coordinates } & \multirow{2}{*}{$\begin{array}{l}\text { Volume } \\
\qquad(\mu \mathrm{l})\end{array}$} & \multirow[b]{2}{*}{ T-score } \\
\hline & & & $\mathrm{x}$ & $\mathrm{y}$ & $\mathrm{z}$ & & \\
\hline \multirow[t]{7}{*}{$\mathrm{TD}$} & Middle occipital gyrus & $\mathrm{R}$ & 29 & 77 & 30 & 6129 & 5.6 \\
\hline & Angular gyrus (25.2) & & & & & & \\
\hline & Inferior parietal lobe (24.1) & & & & & & \\
\hline & Middle occipital gyrus (22.1) & & & & & & \\
\hline & Superior occipital gyrus (11.8) & & & & & & \\
\hline & Superior parietal lobe (9.2) & & & & & & \\
\hline & Cerebellum & $\mathrm{R}$ & 26 & -47 & -25 & 2646 & 5.3 \\
\hline $\mathrm{ASD}>\mathrm{TD}$ & Inferior frontal gyrus & $\mathrm{R}$ & 56 & 26 & -1 & 783 & 4.9 \\
\hline
\end{tabular}

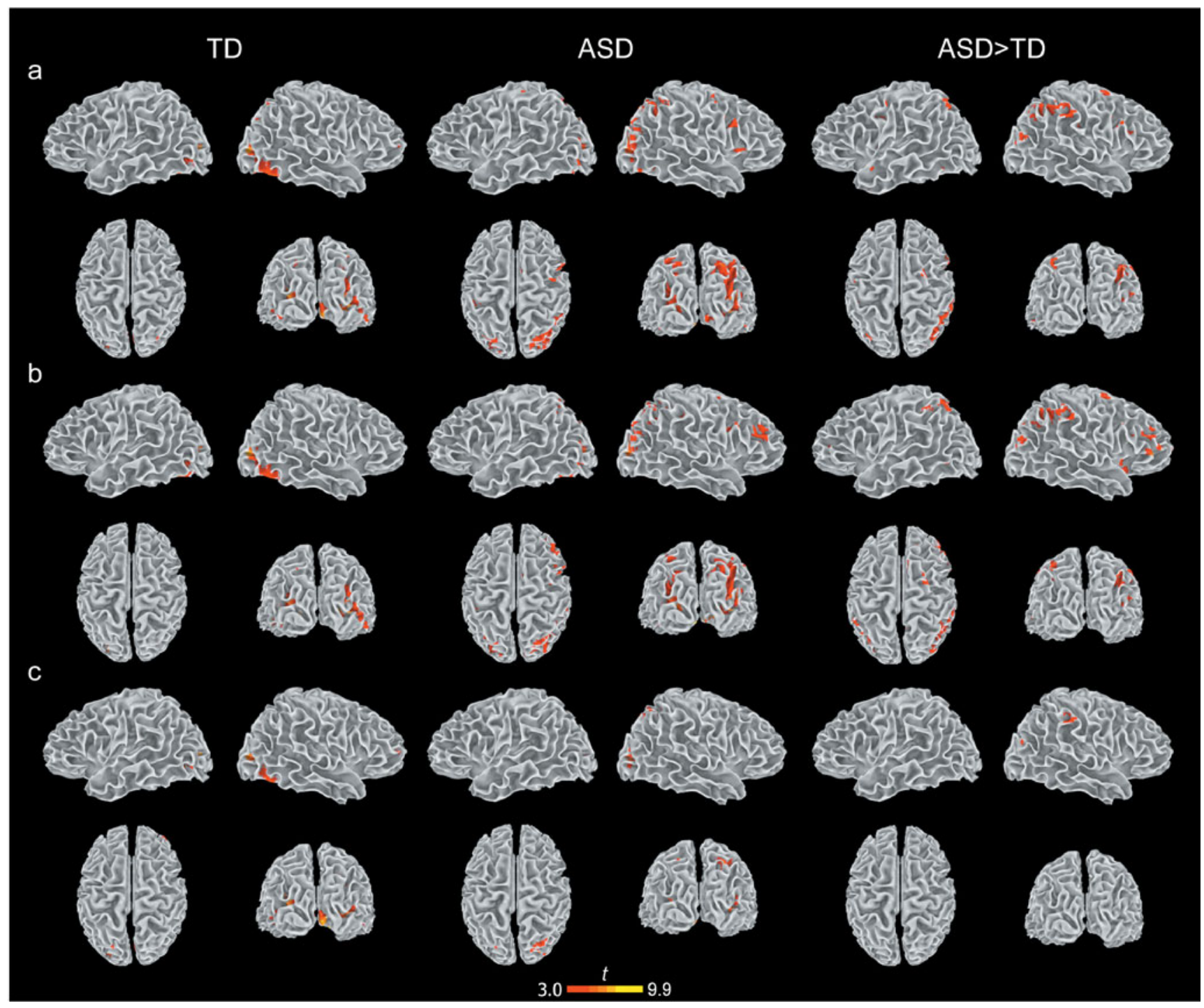

Fig. 5. Significant activation clusters for within group comparisons and clusters showing significant effects on direct group comparisons (ASD > TD) for combined homogeneous and heterogeneous (a), homogeneous (b), and heterogeneous (c) trials. 
Table 3. fMRI BOLD activation for homogeneous and heterogeneous trials versus baseline trials

\begin{tabular}{|c|c|c|c|c|c|c|c|}
\hline & \multirow{2}{*}{$\begin{array}{c}\text { Peak location } \\
\text { Additional regions } \\
(\% \text { volume of cluster })\end{array}$} & \multirow[b]{2}{*}{ Hemisphere } & \multicolumn{3}{|c|}{ Talairach coordinates } & \multirow{2}{*}{$\begin{array}{l}\text { Volume } \\
\qquad(\mu 1)\end{array}$} & \multirow[b]{2}{*}{ T-scor } \\
\hline & & & $\mathrm{x}$ & $\mathrm{y}$ & $\mathrm{z}$ & & \\
\hline \multirow[t]{9}{*}{ TD } & Middle occipital gyrus & $\mathrm{R}$ & 26 & -80 & 6 & 37935 & 10.5 \\
\hline & R. middle occipital gyrus (7.5) & & & & & & \\
\hline & R. fusiform gyrus (7.4) & & & & & & \\
\hline & R. lingual gyrus (6.7) & & & & & & \\
\hline & L. fusiform gyrus (6.4) & & & & & & \\
\hline & L. middle occipital gyrus (6.2) & & & & & & \\
\hline & L. lingual gyrus $(5.5)$ & & & & & & \\
\hline & R. calcarine Fissure (5.4) & & & & & & \\
\hline & Thalamus & $\mathrm{R}$ & 17 & -29 & -1 & 594 & 4.7 \\
\hline \multirow[t]{25}{*}{ ASD } & Middle occipital gyrus & $\mathrm{R}$ & 35 & 73 & 14 & 21546 & 12.7 \\
\hline & Middle occipital gyrus (24.7) & & & & & & \\
\hline & Superior occipital gyrus (14.4) & & & & & & \\
\hline & Superior parietal lobe (10.3) & & & & & & \\
\hline & Angular gyrus (10.0) & & & & & & \\
\hline & Calcarine fissure (8.3) & & & & & & \\
\hline & Inferior parietal lobe (7.6) & & & & & & \\
\hline & Middle occipital gyrus & $\mathrm{L}$ & -26 & 77 & 14 & 15660 & 11.7 \\
\hline & Middle occipital gyrus (29.8) & & & & & & \\
\hline & Superior parietal lobe (16.0) & & & & & & \\
\hline & Superior occipital gyrus (10.2) & & & & & & \\
\hline & Calcarine fissure $(9.3)$ & & & & & & \\
\hline & Fusiform gyrus (6.6) & & & & & & \\
\hline & Inferior occipital gyrus (5.1) & & & & & & \\
\hline & Insula & $\mathrm{R}$ & 35 & 14 & 12 & 5400 & 9.7 \\
\hline & Insula (25.6) & & & & & & \\
\hline & Inferior frontal gyrus (24.7) & & & & & & \\
\hline & Precentral gyrus $(9.7)$ & & & & & & \\
\hline & Middle frontal gyrus & $\mathrm{R}$ & 8 & 2 & 57 & 4995 & 8.3 \\
\hline & Thalamus & $\mathrm{L}$ & -23 & -29 & 3 & 3537 & 11.8 \\
\hline & Caudate nucleus & $\mathrm{R}$ & 20 & -8 & 24 & 2592 & 6.9 \\
\hline & Caudate nucleus & $\mathrm{L}$ & -20 & -29 & 18 & 2241 & 6.9 \\
\hline & Precentral gyrus & $\mathrm{R}$ & 35 & -11 & 51 & 1863 & 7.6 \\
\hline & Cerebelllar vermis & $\mathrm{R}$ & 5 & -65 & -10 & 1026 & 8.4 \\
\hline & Postcentral gyrus & $\mathrm{L}$ & -38 & -32 & 54 & 567 & 5.4 \\
\hline \multirow[t]{14}{*}{$\mathrm{ASD}>\mathrm{TD}$} & Middle occipital gyrus & $\mathrm{R}$ & 35 & -68 & 33 & 8242 & 5.1 \\
\hline & Angular gyrus (30.1) & & & & & & \\
\hline & Inferior parietal lobe (27.6) & & & & & & \\
\hline & Supramarginal gyrus (22.8) & & & & & & \\
\hline & Middle occipital gyrus (7.7) & & & & & & \\
\hline & Precuneus & $\mathrm{R}$ & 8 & -44 & 54 & 3753 & 4.5 \\
\hline & Supplementary Motor Area & $\mathrm{L}$ & -11 & -23 & 48 & 1890 & 3.9 \\
\hline & Inferior frontal gyrus & $\mathrm{R}$ & 35 & 16 & 9 & 1080 & 4.7 \\
\hline & Superior parietal lobe & $\mathrm{L}$ & -32 & -65 & 48 & 945 & 4.5 \\
\hline & Inferior frontal gyrus & $\mathrm{R}$ & 50 & 2 & 24 & 864 & 4.6 \\
\hline & Supplementary Motor Area & $\mathrm{L}$ & -8 & -11 & 60 & 864 & 4.9 \\
\hline & Precentral gyrus & $\mathrm{L}$ & -47 & -5 & 33 & 810 & 4.5 \\
\hline & Inferior frontal gyrus & $\mathrm{R}$ & 50 & 20 & 21 & 594 & 4.2 \\
\hline & Middle occipital gyrus & $\mathrm{R}$ & 44 & -77 & 18 & 513 & 5.5 \\
\hline
\end{tabular}

\section{Baseline trials versus fixation}

Table 2 summarizes cluster corrected regions that showed significant BOLD activity for baseline target absent and present trials compared with fixation. For clusters with volume $>5000 \mu \mathrm{l}$, subregions are listed as the percentage of total cluster volume. Subregions are contiguous areas of cluster activation that extend beyond the peak activation (Eickhoff et al., 2007). TD individuals showed a large cluster of activation, with peak in right middle occipital gyrus, and extending dorsally into inferior and superior parietal lobe. ASD participants showed no significant activation for 
baseline versus fixation comparisons. Activation in left primary motor cortex was seen in both groups, but did not survive cluster correction. Direct group comparison revealed greater activation in ASD than TD group in right inferior frontal gyrus.

\section{All search trials (homogeneous and heterogeneous)}

Figure 5a depicts cluster corrected regions that showed significant BOLD activity for all search trials (homogeneous and heterogeneous combined) compared with baseline trials (listed in Table 3). The TD group showed a large occipital activation cluster, which extended anteriorly into the fusiform gyrus. In the ASD group, similar regions of the occipital lobe were activated, with peaks in the middle occipital gyri; however, activation extended dorsally to the right inferior and bilateral superior parietal lobes. In addition, the ASD group recruited frontal cortex within the right inferior and middle frontal gyri. Direct group comparisons of all search trials versus baseline revealed significantly greater activation for the ASD group in frontal regions including the right

Table 4. fMRI BOLD activation for homogeneous trials versus baseline trials

\begin{tabular}{|c|c|c|c|c|c|c|c|}
\hline & \multirow{2}{*}{$\begin{array}{c}\text { Peak location } \\
\text { Additional regions } \\
(\% \text { volume of cluster })\end{array}$} & \multirow[b]{2}{*}{ Hemisphere } & \multicolumn{3}{|c|}{ Talairach coordinates } & \multirow{2}{*}{$\begin{array}{l}\text { Volume } \\
\qquad(\mu \mathrm{l})\end{array}$} & \multirow[b]{2}{*}{ T-score } \\
\hline & & & $\mathrm{x}$ & $\mathrm{y}$ & $\mathrm{z}$ & & \\
\hline \multirow[t]{15}{*}{$\mathrm{TD}$} & Middle occipital gyrus & $\mathrm{R}$ & 26 & -80 & 6 & 15579 & 8.8 \\
\hline & Middle occipital gyrus (20.2) & & & & & & \\
\hline & Fusiform gyrus (15.7) & & & & & & \\
\hline & Inferior temporal gyrus (11.4) & & & & & & \\
\hline & Inferior occipital gyrus (10.8) & & & & & & \\
\hline & Cerebellum (VI) (6.8) & & & & & & \\
\hline & Superior occipital gyrus (6.5) & & & & & & \\
\hline & Cerebellum (Crus I) (5.9) & & & & & & \\
\hline & Middle occipital gyrus & $\mathrm{L}$ & -29 & -77 & 9 & 8937 & 8.8 \\
\hline & Middle occipital gyrus (22.5) & & & & & & \\
\hline & Fusiform gyrus (21.1) & & & & & & \\
\hline & Inferior occipital gyrus (17.9) & & & & & & \\
\hline & Lingual gyrus (9.2) & & & & & & \\
\hline & Cerebellum (Crus I) (7.7) & & & & & & \\
\hline & Cerebellum (VI) (6.8) & & & & & & \\
\hline \multirow[t]{14}{*}{ ASD } & Inferior occipital gyrus & $\mathrm{L}$ & -29 & -68 & -4 & 26757 & 11.8 \\
\hline & R. middle occipital gyrus (18.6) & & & & & & \\
\hline & L. middle occipital gyrus (18.4) & & & & & & \\
\hline & R. superior occipital gyrus (10.8) & & & & & & \\
\hline & L. superior occipital gyrus (6.0) & & & & & & \\
\hline & Supplementary Motor Area & $\mathrm{L}$ & -5 & 5 & 45 & 3240 & 7.7 \\
\hline & Inferior frontal gyrus & $\mathrm{R}$ & 47 & 35 & 21 & 2592 & 6.1 \\
\hline & Superior parietal lobe & $\mathrm{L}$ & -23 & -62 & 48 & 1944 & 8.6 \\
\hline & Supplementary Motor Area & $\mathrm{L}$ & -11 & -14 & 57 & 1647 & 6.1 \\
\hline & Caudate & $\mathrm{L}$ & -17 & 2 & 27 & 1458 & 10.0 \\
\hline & Precentral gyrus & $\mathrm{R}$ & 32 & -8 & 48 & 1107 & 5.7 \\
\hline & Inferior frontal gyrus & $\mathrm{R}$ & 44 & 5 & 24 & 621 & 4.9 \\
\hline & Inferior parietal lobe & $\mathrm{L}$ & -35 & -50 & 36 & 621 & 6.2 \\
\hline & Inferior parietal lobe & $\mathrm{L}$ & -44 & -38 & 42 & 594 & 4.8 \\
\hline \multirow[t]{14}{*}{$\mathrm{ASD}>\mathrm{TD}$} & Middle occipital gyrus & $\mathrm{R}$ & 35 & -68 & 33 & 6642 & 4.6 \\
\hline & Angular gyrus (31.7) & & & & & & \\
\hline & Inferior parietal lobe (28.6) & & & & & & \\
\hline & Supramarginal gyrus (14.8) & & & & & & \\
\hline & Middle occipital gyrus (10.5) & & & & & & \\
\hline & Precentral gyrus & $\mathrm{R}$ & 23 & -14 & 45 & 3564 & 4.0 \\
\hline & Supplementary motor area & $\mathrm{L}$ & -8 & -14 & 57 & 3375 & 4.5 \\
\hline & Supplementary motor area & $\mathrm{R}$ & 5 & 8 & 54 & 1863 & 3.9 \\
\hline & Inferior frontal gyrus & $\mathrm{R}$ & 23 & 17 & -13 & 1755 & 4.1 \\
\hline & Inferior parietal lobe & $\mathrm{L}$ & -41 & -47 & 42 & 1620 & 4.2 \\
\hline & Superior frontal gyrus & $\mathrm{L}$ & -11 & -2 & 45 & 1485 & 5.4 \\
\hline & Precuneus & $\mathrm{L}$ & -2 & -47 & 51 & 1134 & 4.3 \\
\hline & Inferior frontal gyrus & $\mathrm{R}$ & 50 & 35 & 21 & 891 & 4.2 \\
\hline & Inferior parietal lobe & $\mathrm{L}$ & -38 & -59 & 54 & 648 & 4.1 \\
\hline
\end{tabular}


inferior frontal gyrus. The ASD group also showed greater activation in the superior parietal lobe, as well as in a large cluster with peak in the right middle occipital gyrus that extended into the inferior parietal lobe. For the comparison of search trials with fixation, there was greater activation of the right superior frontal gyrus $(\mathrm{x}=20, \mathrm{y}=-5, \mathrm{z}=51 ; t=$ 4.2) in the ASD as compared to the TD group. No inverse effects of greater activity in the TD group were detected.

\section{Homogeneous search trials}

Figure $5 \mathrm{~b}$ depicts cluster corrected regions that showed significant BOLD activity for homogeneous search trials compared with baseline (listed in Table 4). A large cluster of significant BOLD activity for the TD group was located in occipitotemporal cortex, with a peak located in the middle occipital gyrus. The ASD group showed a more distributed pattern of activation, with significant clusters appearing in right inferior frontal gyrus, left inferior and superior parietal lobe, and bilateral occipital cortex. The ASD group also displayed activation in the supplementary motor area bilaterally, extending into the left superior frontal gyrus. A direct group comparison revealed significantly greater frontal activation for the ASD group, including the left superior frontal and right inferior frontal gyri. Additionally, the ASD group showed greater activation than the TD group in bilateral supplementary motor cortices and inferior parietal lobule, and right middle occipital gyrus. For the comparison of homogeneous search trials with fixation, greater activation in the ASD as compared to the TD group was also found in right superior frontal gyrus $(\mathrm{x}=23, \mathrm{y}=2, \mathrm{z}=60 ; t=4.5)$. No inverse effects of greater activity in the TD group were detected.

\section{Heterogeneous search trials}

Figure $5 \mathrm{c}$ depicts cluster corrected regions that showed significant BOLD activity for heterogeneous search trials compared with baseline (listed in Table 5). Peak activation for
TD participants was located in left occipital cortex, with a cluster extending bilaterally in the occipital lobe and into the right fusiform gyrus, and in right superior occipital and inferior frontal gyri. Peak activation for the ASD group was located in the right superior parietal lobe and right middle occipital gyrus. Direct group comparisons for heterogeneous versus baseline revealed no significant effects. However, group comparison for heterogeneous search versus fixation revealed greater activation for the ASD group in right superior $(\mathrm{x}=20, \mathrm{y}=-8, \mathrm{z}=51 ; t=4.0)$ and inferior $(\mathrm{x}=44, \mathrm{y}=2, \mathrm{z}=15 ; t=4.4)$ frontal gyri, middle temporal gyrus $(\mathrm{x}=47, \mathrm{y}=-47, \mathrm{z}=-1 ; t=4.0)$, and areas of the occipital lobe $(\mathrm{x}=32, \mathrm{y}=-68, \mathrm{z}=33 ; t=4.1)$. No inverse effects of greater activity in the TD group were detected.

\section{Heterogeneous versus homogeneous trials}

Pair-wise comparisons were conducted for each group to examine the differences between homogeneous and heterogeneous search trials (Table 6). Greater activation for homogeneous compared with heterogeneous trials in TD participants was observed in the right temporal pole and the left postcentral gyrus, extending into the inferior parietal lobule. Inverse effects (activation greater in heterogeneous compared with homogeneous trials) in TD individuals were detected in bilateral occipital lobe and right supplementary motor area, extending into the cingulate gyrus. Pair-wise comparisons for individuals with ASD revealed no significant differences between the two conditions.

\section{Correlations with performance and diagnostic measures}

These exploratory analyses were performed to examine whether brain activation was related to behavioral measures. Regions of interest (ROI) were created from clusters of activation from all trials versus baseline for all participants, and included right inferior frontal gyrus (rIFG), right

Table 5. fMRI BOLD activation to heterogeneous trials versus baseline trials

\begin{tabular}{|c|c|c|c|c|c|c|c|}
\hline & \multirow{2}{*}{$\begin{array}{c}\text { Peak location } \\
\text { Additional regions } \\
(\% \text { volume of cluster })\end{array}$} & \multirow[b]{2}{*}{ Hemisphere } & \multicolumn{3}{|c|}{ Talairach coordinates } & \multirow{2}{*}{$\begin{array}{l}\text { Volume } \\
\qquad(\mu \mathrm{l})\end{array}$} & \multirow[b]{2}{*}{ T-score } \\
\hline & & & $\mathrm{x}$ & $\mathrm{y}$ & $\mathrm{z}$ & & \\
\hline \multirow[t]{10}{*}{ TD } & Middle occipital gyrus & $\mathrm{L}$ & -29 & -71 & -13 & 24408 & 11.6 \\
\hline & R. lingual gyrus (10.2) & & & & & & \\
\hline & L. calcarine fissure (8.5) & & & & & & \\
\hline & R. fusiform gyrus (8.2) & & & & & & \\
\hline & L. lingual gyrus (8.1) & & & & & & \\
\hline & R. inferior occipital gyrus (6.0) & & & & & & \\
\hline & L. middle occipital gyrus (5.9) & & & & & & \\
\hline & R. calcarine fissure (5.9) & & & & & & \\
\hline & Superior occipital gyrus & $\mathrm{R}$ & 29 & -71 & 30 & 783 & 4.6 \\
\hline & Inferior frontal gyrus & $\mathrm{R}$ & 34 & 23 & 9 & 567 & 4.6 \\
\hline \multirow[t]{2}{*}{ ASD } & Superior parietal lobe & $\mathrm{R}$ & 23 & -68 & 45 & 2619 & 9.2 \\
\hline & Middle occipital gyrus & $\mathrm{R}$ & 32 & -86 & 3 & 513 & 5.9 \\
\hline
\end{tabular}


Table 6. fMRI BOLD activation to homogeneous versus heterogeneous trials

\begin{tabular}{|c|c|c|c|c|c|c|c|}
\hline & \multirow[b]{2}{*}{ Peak location } & \multirow[b]{2}{*}{ Hemisphere } & \multicolumn{3}{|c|}{ Talairach coordinates } & \multirow{2}{*}{$\begin{array}{l}\text { Volume } \\
\qquad(\mu \mathrm{l})\end{array}$} & \multirow[b]{2}{*}{ T-score } \\
\hline & & & $\mathrm{x}$ & $\mathrm{y}$ & $\mathrm{z}$ & & \\
\hline \multirow[t]{4}{*}{$\mathrm{TD}-\mathrm{HOM}>\mathrm{HET}$} & Temporal pole & $\mathrm{R}$ & 56 & 2 & 3 & 2376 & 5.7 \\
\hline & Lentiform nucleus & $\mathrm{L}$ & -17 & -2 & -7 & 1215 & 4.3 \\
\hline & Caudate & $\mathrm{R}$ & 35 & -14 & -7 & 1107 & 4.2 \\
\hline & Postcentral gyrus & $\mathrm{L}$ & -56 & -26 & 24 & 837 & 4.9 \\
\hline \multirow[t]{3}{*}{$\mathrm{TD}-\mathrm{HET}>\mathrm{HOM}$} & Supplementary Motor Area & $\mathrm{R}$ & 2 & 11 & 51 & 1890 & 4.6 \\
\hline & Lingual gyrus & $\mathrm{R}$ & 20 & -74 & -10 & 918 & 4.2 \\
\hline & Calcarine fissure & $\mathrm{L}$ & -14 & -71 & 9 & 783 & 5.2 \\
\hline
\end{tabular}

posterior parietal cortex, and left and right middle occipital gyri. Average $Z$-scores for each ROI were computed for comparisons of homogeneous versus baseline trials and heterogeneous versus baseline trials, and then correlated with median RT, slope, y-intercept, and error rates. The RT slope for heterogeneous target present trials was significantly correlated with average $Z$-score of the rIFG ROI in the ASD group, $r(7)=-.71 ; p<.05$, but not the TD group, $r(11)=$ $-.03 ; p>.9$, suggesting that increased search efficiency was associated with greater inferior frontal activation in the ASD (but not the TD) group. Error rates for homogeneous trials were negatively correlated with IFG ROI average $Z$-score for homogeneous trials in the ASD group, $r(7)=$ $-.72 ; p<.05$, but not the TD group, $r(11)=-.11 ; p>.7$. Conversely, error rates for heterogeneous trials were positively correlated with rIFG ROI average $Z$-score for heterogeneous trials in the TD group, $r(11)=.73 ; p<.01$, but not the ASD group, $r(7)=-.02 ; p>$.9. All other correlations were nonsignificant, $p \mathrm{~s}>.1$.

\section{DISCUSSION}

Our behavioral findings showed the expected effects of target composition, with a significantly greater number of errors and longer RTs for heterogeneous (compared with homogeneous) trials in both groups. Contrary to our expectation, we did not find a main effect of group for either error rates or RT. However, we detected a marginally significant interaction between group and search difficulty, as individuals with ASD were faster than TD individuals in the hard (heterogeneous), but slower in the easy (homogeneous) search condition. This is consistent with previous findings suggesting that individuals with ASD excel in more difficult search tasks (e.g., O'Riordan et al., 2001). Furthermore, RT by set size slopes, were significantly shallower for the ASD group, indicating that participants with ASD were affected to a lesser extent by larger set sizes. While slopes for homogeneous trials did not meet strict criteria for efficient, parallel processing $(<10 \mathrm{~ms} /$ item $)$, they were significantly shallower compared with heterogeneous slopes in TD individuals, indicating that search efficiency was reduced in heterogeneous compared with homogeneous search conditions. Interestingly, individuals with ASD did not demon- strate significant differences in slope for homogeneous compared with heterogeneous distractors, indicating that search efficiency was not affected by distractor composition in ASD.

The unexpected absence of a main effect of group may be explained by the marginally longer RTs on baseline target present trials in the ASD group, probably reflecting slower basic visuomotor coordination. Previous studies have shown that individuals with ASD demonstrate atypical motor preparation (Rinehart et al., 2001), but intact visual information processing (Scheuffgen et al., 2000) compared with TD individuals. This suggests that slowed visuomotor coordination may account for the unexpectedly longer RTs in the ASD group for efficient search (homogeneous distractors), whereas enhanced visual search abilities may offset slowed visuomotor coordination on the more demanding inefficient search conditions (heterogeneous distractors).

Patterns of brain activation for both efficient and inefficient visual search differed between ASD and TD groups. In the TD group, activation for all search trials (homogeneous and heterogeneous combined) was found in a large contiguous cluster in posterior regions, peaking in occipital cortex and extending into the inferior temporal lobe. In the ASD group, a more distributed network of brain areas was activated, which extended beyond bilateral occipital cortex into more dorsal regions, such as the precuneus and superior parietal lobe, and also included right frontal cortices. Similar overall patterns of within-group activation effects were found when efficient and inefficient (homogeneous, heterogeneous) search trials were examined separately, with activity in frontal-parietal regions in the ASD, but not the TD group. Pair-wise comparisons revealed differences in activation for homogeneous versus heterogeneous search trials in the TD group, but not in the ASD group. Specifically, TD individuals recruited bilateral occipital and right frontal areas to a greater extent in heterogeneous versus homogeneous trials. Absence of differences in the ASD group, along with similar slopes for both homogeneous and heterogeneous conditions suggests that behavioral performance and neural recruitment were similar for both efficient and inefficient search tasks in ASD participants. 
Direct group comparisons of homogeneous and heterogeneous trials (both separately and combined) support the finding of overall more extensive activation in the ASD group. Consistent with our initial hypothesis, activation in the ASD group was significantly greater in occipito-temporal regions. However unexpectedly, the ASD also showed greater activation in frontal and parietal regions.

In agreement with previous reports investigating visuospatial strengths (Lee et al., 2007; Manjaly et al., 2007; Ring et al., 1999), we found that individuals with ASD display increased activation of occipital regions. These findings are consistent with the hypothesis that superior visual search abilities in individuals with ASD are the result of enhanced discrimination. As predicted by Caron and colleagues (2006) enhanced activation of early visual areas, as evidenced by our ASD group, may contribute to superior visuospatial abilities in autism. This finding is accordance with the enhanced perceptual functioning model (Mottron et al., 2006) and adds to the existing literature demonstrating atypically enhanced activation of visual cortices in ASD for other types of task, such as sentence comprehension (Kana et al., 2006), semantic decision (Gaffrey et al., 2007), and verbal working memory (Koshino et al., 2005).

Contrary to previous reports, we found that individuals with ASD evidenced increased frontoparietal activation compared with TD individuals. These effects were seen for both homogeneous and heterogeneous search conditions. Activated areas, which included superior and inferior parietal lobe and superior and inferior frontal gyri, form a functional network responsible for top-down biasing of visual attention (Kastner \& Ungerleider, 2000). In conflict with our original hypothesis, our findings suggest accelerated search performance by individuals with ASD may be related to increased activation in areas associated with top-down, in addition to bottom-up, control of visual attention. Moreover, correlations between inferior frontal activity and RT by set size slope for heterogeneous present trials demonstrated that increased activation of rIFG was inversely correlated with RT by set size slopes for heterogeneous (target present) trials in the ASD, but not the TD, group. This suggests that increased search efficiency in the ASD group may be related to increased top-down control modulated by inferior frontal regions.

Using repetitive transcranial magnetic stimulation, Muggleton et al. (2003) found that stimulation to the FEFs impaired inefficient conjunctive search, but not efficient feature search. Greater activation in superior frontal gyrus as detected in our study (search task vs. fixation comparison) was consistent with the FEF, with a peak detected within a few millimeters of activation peaks previously identified by Donner et al. (2002) and Shulman et al. (2003). Our finding of increased recruitment of FEF by ASD as compared with TD individuals suggests that the FEF may also serve to enhance search performance in individuals with ASD.

Our findings of enhanced frontoparietal activation stand in contrast to previous fMRI investigations of EFT perfor- mance in individuals with ASD. This may be attributed to differences between the EFT and the search paradigm implemented here. First, there are perceptual differences between locating a target embedded within a complex figure and locating a target within an array of separate distractors. Second, although both tasks require target-related search, target and distractors with our search paradigm remain constant while targets and complex figures vary with each trial in an EFT. This results in enhanced top-down modulation of target and distractor features by means of both positive and negative priming of target and distractor features.

O'Riordan (2000) reported no difference in positive and negative priming effects during visual search between ASD and TD individuals, which suggests that top-down modulation of object-based representations is at least intact in individuals with ASD. Top-down modulation of visual attention can influence stimulus processing by enhancing responses for attended stimuli, filtering irrelevant information, and increasing salience of stimulus features; it is dependent on feedback projections from frontal-parietal areas to visual cortices (Kastner \& Ungerleider, 2000). While our finding of enhanced frontal recruitment during visual search in ASD may be consistent with enhanced top-down control of visual attention, it is surprising given previous theories of reduced prefrontal control in autism (Minshew et al., 2002). It also raises new questions regarding a model of generally reduced long distance connectivity in ASD (Just et al., 2004). Although our study did not include analyses of functional connectivity, combined participation of frontal and occipital regions during visual search suggests that cooperation between distal regions may be task-dependent, rather than generally deficient in ASD. Functional connectivity between primary visual cortex and inferior frontal cortex has been found to be reduced in autism during visuomotor coordination in the absence of a search task (Villalobos et al., 2005), whereas the present findings suggest that during visual search, prefrontal cortex including inferior frontal lobe may play an unusually enhanced role in facilitating efficient visual search in individuals with ASD.

The interpretation of our current results remains limited given a sample size that was reduced by severe motion in several children with ASD. In addition, we did not observe accelerated RT in the ASD group, similar to previous fMRI reports investigating visuospatial tasks (Lee et al., 2007; Ring et al., 1999). Such null findings may be related to the unique testing environment or relatively small samples included in functional imaging studies.

While our findings should be interpreted with some caution due to our small sample, they suggest a differential pattern of activation in individuals with ASD as compared to TD individuals. In particular, children with ASD recruited a network including frontal, parietal, and occipital cortices, whereas activation in a matched TD comparison group was less extensive and primarily limited to occipito-temporal regions. In agreement with the hypothesis that enhanced discrimination underlies superior visual search abilities in ASD we found increased occipital activation in ASD com- 
pared with TD individuals. Additionally, our results suggest that accelerated performance in individuals with ASD, particularly increased search efficiency, may be related to enhanced top-down modulation of visual attention.

\section{ACKNOWLEDGMENTS}

Supported by the National Institute on Deafness and Other Communicative Disorders R01-DC006155 and 1T32 DC007361-03 (BK). Special thanks to Frank Haist for technical assistance, to Natacha Akshoomoff for help in the recruitment and diagnosis of participants with autism, and especially to the children and families who generously participated.

\section{REFERENCES}

American Psychiatric Association. (2000). Diagnostic and Statistical Manual of Mental Disorders (4th ed.-Text Revision). Washington, DC: American Psychiatric Association Press.

Anderson, E.J., Mannan, S.K., Husain, M., Rees, G., Sumner, P., Mort, D.J., McRobbie, D., \& Kennard, C. (2007). Involvement of prefrontal cortex in visual search. Experimental Brain Research, 180, 289-302.

Booth, J.R., Burman, D.D., Meyer, J.R., Lei, Z., Trommer, B.L., Davenport, N.D., Li, W., Parrish, TB., Gitelman, D.R., \& Mesulam, M.M. (2003). Neural development of selective attention and response inhibition. Neuroimage, 20, 737-751.

Caron, M.J., Mottron, L., Berthiaume, C., \& Dawson, M. (2006). Cognitive mechanisms, specificity and neural underpinnings of visuospatial peaks in autism. Brain, 129(Pt 7), 1789-1802.

Corbetta, M. \& Shulman, G.L. (2002). Control of goal-directed and stimulus-driven attention in the brain. Nature Reviews Neuroscience, 3, 201-215.

Cox, R.W. (1996). AFNI: Software for analysis and visualization of functional magnetic resonance neuroimages. Computers and Biomedical Research, 29, 162-173.

Dakin, S. \& Frith, U. (2005). Vagaries of visual perception in autism. Neuron, 48, 497-507.

Donner, T., Kettermann, A., Diesch, E., Ostendorf, F., Villringer, A., \& Brandt, S.A. (2000). Involvement of the human frontal eye field and multiple parietal areas in covert visual selection during conjunction search. European Journal of Neuroscience, 12, 3407-3414.

Donner, T.H., Kettermann, A., Diesch, E., Ostendorf, F., Villringer, A., \& Brandt, S.A. (2002). Visual feature and conjunction searches of equal difficulty engage only partially overlapping frontoparietal networks. Neuroimage, 15, 16-25.

Duncan, J. \& Humphreys, G.W. (1989). Visual search and stimulus similarity. Psychological Review, 96, 433-458.

Eickhoff, S.B., Paus, T., Caspers, S., Grosbras, M.H., Evans, A.C., Zilles, K., \& Amunts, K. (2007). Assignment of functional activations to probabilistic cytoarchitectonic areas revisited. Neuroimage, 36, 511-521.

Everts, R., Lidzba, K., Wilke, M., Kiefer, C., Mordasini, M., Schroth, G., Perrig, W., \& Steinlin, M. (2008). Strengthening of laterality of verbal and visuospatial functions during childhood and adolescence. Human Brain Mapping, [Epub ahead of print].

Forman, S.D., Cohen, J.D., Fitzgerald, M., Eddy, W.F., Mintun, M.A., \& Noll, D.C. (1995). Improved assessment of significant activation in functional magnetic resonance imaging
(fMRI): Use of a cluster-size threshold. Magnetic Resonance Medicine, 33, 636-647.

Gaffrey, M.S., Kleinhans, N.M., Haist, F., Akshoomoff, N., Campbell, A., Courchesne, E., \& Müller, R.A. (2007). Atypical brain organization for lexical semantic decision in autism spectrum disorders: fMRI evidence of increased visual imagery. Neuropsychologia, 45, 1672-1684.

Gitelman, D.R., Parrish, T.B., Friston, K.J., \& Mesulam, M.M. (2002). Functional anatomy of visual search: Regional segregations within the frontal eye fields and effective connectivity of the superior colliculus. Neuroimage, 15, 970-982.

Jarrold, C., Gilchrist, I.D., \& Bender, A. (2005). Embedded figures detection in autism and typical development: Preliminary evidence of a double dissociation in relationships with visual search. Developmental Science, 8, 344-351.

Jolliffe, T. \& Baron-Cohen, S. (1997). Are people with autism and Asperger syndrome faster than normal on the embedded figures test? Journal of Child Psychology and Psychiatry, 38, 527-534.

Just, M.A., Cherkassky, V.L., Keller, T.A., \& Minshew, N.J. (2004). Cortical activation and synchronization during sentence comprehension in high-functioning autism: Evidence of underconnectivity. Brain, 127(Pt 8), 1811-1821.

Kana, R.K., Keller, T.A., Cherkassky, V.L., Minshew, N.J., \& Just, M.A. (2006). Sentence comprehension in autism: Thinking in pictures with decreased functional connectivity. Brain, 129 (Pt 9), 2484-2493.

Kastner, S. \& Ungerleider, L.G. (2000). Mechanisms of visual attention in the human cortex. Annual Review of Neuroscience, 23, 315-341.

Koshino, H., Carpenter, P.A., Minshew, N.J., Cherkassky, V.L., Keller, T.A., \& Just, M.A. (2005). Functional connectivity in an fMRI working memory task in high-functioning autism. Neuroimage, 24, 810-821.

Lee, P.S., Foss-Feig, J., Henderson, J.G., Kenworthy, L.E., Gilotty, L., Gaillard, W.D., \& Vaidya, C.J. (2007). Atypical neural substrates of embedded figures task performance in children with autism spectrum disorder. Neuroimage, 38, 184-193.

Leonards, U., Sunaert, S., Van Hecke, P., \& Orban, G.A. (2000). Attention mechanisms in visual search-An fMRI study. Journal of Cognitive Neuroscience, 12 (Suppl 2), 61-75.

Lord, C., Rutter, M., DiLavore, P.C., \& Risi, S. (1999). Autism Diagnostic Observation Schedule-WPS (ADOS-WPS). Los Angeles, CA: Western Psychological Services.

Manjaly, Z.M., Bruning, N., Neufang, S., Stephan, K.E., Brieber, S., Marshall, J.C., Kamp-Becker, I., Remschmidt, H., HerpertzDahlmann, B., Konrad, K., \& Fink, G.R. (2007). Neurophysiological correlates of relatively enhanced local visual search in autistic adolescents. Neuroimage, 35, 283-291.

Minshew, N.J., Sweeney, J., \& Luna, B. (2002). Autism as a selective disorder of complex information processing and underdevelopment of neocortical systems. Molecular Psychiatry, 7(Suppl 2), S14-S15.

Morgan, B., Maybery, M., \& Durkin, K. (2003). Weak central coherence, poor joint attention, and low verbal ability: Independent deficits in early autism. Developmental Psychology, 39, 646-656.

Mottron, L., Dawson, M., Soulieres, I., Hubert, B., \& Burack, J. (2006). Enhanced perceptual functioning in autism: An update, and eight principles of autistic perception. Journal of Autism and Developmental Disorders, 36, 27-43.

Muggleton, N.G., Juan, C.H., Cowey, A., \& Walsh, V. (2003). Human frontal eye fields and visual search. Journal of Neurophysiology, 89, 3340-3343. 
Müller, N.G., Donner, T.H., Bartelt, O.A., Brandt, S.A., Villringer, A., \& Kleinschmidt, A. (2003). The functional neuroanatomy of visual conjunction search: A parametric fMRI study. Neuroimage, 20, 1578-1590.

Nobre, A.C., Coull, J.T., Walsh, V., \& Frith, C.D. (2003). Brain activations during visual search: Contributions of search efficiency versus feature binding. Neuroimage, 18, 91-103.

O'Riordan, M. (2000). Superior modulation of activation levels of stimulus representations does not underlie superior discrimination in autism. Cognition, 77, 81-96.

O'Riordan, M. (2004). Superior visual search in adults with autism. Autism, 8, 229-248.

O'Riordan, M. \& Plaisted, K. (2001). Enhanced discrimination in autism. Quarterly Journal of Experimental Psychology A, 54, 961-979.

O'Riordan, M., Plaisted, K.C., Driver, J., \& Baron-Cohen, S. (2001). Superior visual search in autism. Journal of Experimental Psychology: Human Perception and Performance, 27, 719-730.

Plaisted, K., O’Riordan, M., \& Baron-Cohen, S. (1998). Enhanced visual search for a conjunctive target in autism: A research note. Journal of Child Psychology and Psychiatry, 39, 777-783.

Rinehart, N.J., Bradshaw, J.L., Brereton, A.V., \& Tonge, B.J. (2001). Movement preparation in high-functioning autism and Asperger disorder: A serial choice reaction time task involving motor reprogramming. Journal of Autism and Developmental Disorders, 31, 79-88.

Ring, H.A., Baron-Cohen, S., Wheelwright, S., Williams, S.C., Brammer, M., Andrew, C., \& Bullmore, E.T. (1999). Cerebral correlates of preserved cognitive skills in autism: A functional MRI study of embedded figures task performance. Brain, $122(\mathrm{Pt}$ 7), 1305-1315.
Rutter, M., Le Couteur, A., \& Lord, C. (2003). Autism Diagnostic Interview-Revised. Los Angeles, CA: Western Psychological Services.

Scheuffgen, K., Happe, F., Anderson, M., \& Frith, U. (2000). High "intelligence," low "IQ"? Speed of processing and measured IQ in children with autism. Developmental Psychopathology, 12, 83-90.

Shah, A. \& Frith, U. (1983). An islet of ability in autistic children: A research note. Journal of Child Psychology and Psychiatry, 24, 613-620.

Shah, A. \& Frith, U. (1993). Why do autistic individuals show superior performance on the block design task? Journal of Child Psychology Psychiatry, 34, 1351-1364.

Shulman, G.L., McAvoy, M.P., Cowan, M.C., Astafiev, S.V., Tansy, A.P., d'Avossa, G., \& Corbetta, M. (2003). Quantitative analysis of attention and detection signals during visual search. Journal of Neurophysiology, 90, 3384-3397.

Sternberg, S. (1966). High-speed scanning in human memory. Science, 153, 652-654.

Villalobos, M.E., Mizuno, A., Dahl, B.C., Kemmotsu, N., \& Müller, R.A. (2005). Reduced functional connectivity between V1 and inferior frontal cortex associated with visuomotor performance in autism. Neuroimage, 25, 916-925.

Wechsler, D. (1999). Wechsler's Abbreviated Scale of Intelligence. San Antonio, TX: The Psychological Corporation.

Wilkinson, D.T., Halligan, P.W., Henson, R.N., \& Dolan, R.J. (2002). The effects of interdistracter similarity on search processes in superior parietal cortex. Neuroimage, 15, 611-619.

Wolfe, J.M. (1994). Guided Search 2.0: A revised model of visual search. Psychonomic Bulletin \& Review, 1, 202-238. 